\title{
Can We Use a Brain-Computer Interface and Manipulate a Mouse at the Same Time?
}

\author{
Jonathan Mercier-Ganady* Émilie Loup-Escande ${ }^{\dagger}$ Laurent George $^{\dagger}$ Colomban Busson ${ }^{\dagger}$ Maud Marchal ${ }^{\ddagger}$ Anatole Lécuyer* $^{*}$

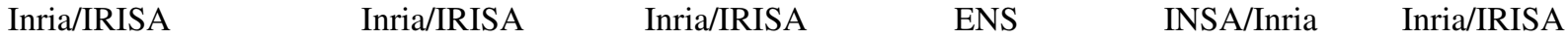

\begin{abstract}
Brain-Computer Interfaces (BCI) introduce a novel way of interacting with real and virtual environments by directly exploiting cerebral activity. However in most setups using a BCI, the user is explicitly asked to remain as motionless as possible, since muscular activity is commonly admitted to add noise and artifacts in brain electrical signals. Thus, as for today, people have been rarely let using other classical input devices such as mice or joysticks simultaneously to a BCI-based interaction. In this paper, we present an experimental study on the influence of manipulating an input device such as a standard computer mouse on the performance of a BCI system. We have designed a 2-class BCI which relies on Alpha brainwaves to discriminate between focused versus relaxed mental activities. The study uses a simple virtual environment inspired by the well-known Pac-Man videogame and based on BCI and mouse controls. The control of mental activity enables to eat pellets in a simple 2D virtual maze. Different levels of motor activity achieved with the mouse are progressively introduced in the gameplay: 1) no motor activity (control condition), 2) a semi-automatic motor activity, and 3) a highly-demanding motor activity. As expected the BCI performance was found to slightly decrease in presence of motor activity. However, we found that the BCI could still be successfully used in all conditions, and that relaxed versus focused mental activities could still be significantly discriminated even in presence of a highly-demanding mouse manipulation. These promising results pave the way to future experimental studies with more complex mental and motor activities, but also to novel 3D interaction paradigms that could mix BCI and other input devices for virtual reality and videogame applications.
\end{abstract}

CR Categories: H.5.2 [Information Interfaces and Presentation]: User Interfaces-Input devices and strategies I.3.7 [Computer Graphics]: Three-Dimensional Graphics and Realism-Virtual reality;

Keywords: brain-computer interface, EEG, motor activity, mental activity

\section{Introduction}

A Brain-Computer Interface (BCI) can measure electrical signals resulting from voluntary or involuntary brain activity in order to transform them into meaningful information or commands for a computerized system [McFarland and Wolpaw 2011]. Most BCI systems rely on ElectroEncephaloGraphy (EEG) headsets to ac-

*e-mail: \{jonathan.mercier-ganady|anatole.lecuyer\}@inria.fr

$\dagger$ e-mail: \{emilie.escande|laurent.f.george|colomban.busson\}@gmail.com

‡e-mail: maud.marchal@irisa.fr quire electrical signals at the level of the head scalp. Up to now, there have been numerous and various attempts made to connect BCI with Virtual Reality (VR) technologies [Lotte et al. 2013]. BCI can be successfully used to interact with a virtual environment (VE) or a videogame in a novel way by directly using mental activity [Allison et al. 2007]. Virtual environments can also be very useful for studying the usage and the learning of BCI systems in safe and perfectly controlled conditions [Lotte et al. 2013].

However, as for today, there have been very few studies that have combined BCI with other and more standard input devices such as mice, joysticks or gamepads [Leeb et al. 2013; Mühl et al. 2010]. One main reason is that muscular activity is known to add artifacts in electrical signals that can considerably impair the process of identifying brain activity. Several approaches have been proposed to detect and remove muscular artifacts, such as Independent Component Analysis or Principal Component Analysis [Lee and Choi 2003]. But these approaches are not always effective. Thus, the $\mathrm{BCI}$ user is often asked to remain motionless and is not let using other interaction devices based on his motor activity. As a result, the integration of BCI with VR technologies, and their combination with other 3D interaction techniques remain at a very early stage.
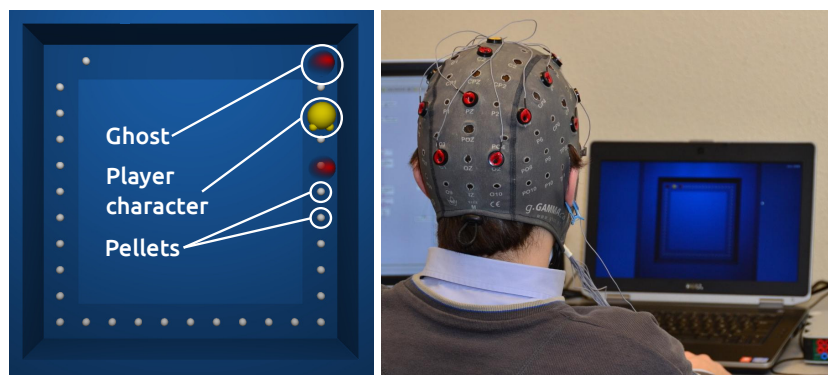

Figure 1: Experimental setup: (Left) Screenshot of our simplified version of Pac-Man videogame; (Right) Participant wearing EEG cap in front of monitor screen.

This paper aims at studying if a simple BCI could be used, in the context of interacting with a VE, in combination with a mouse manipulation, and with successive and progressive levels of motor activity. To do so, we have designed a VE using a BCI that relies on Alpha brainwaves to discriminate between two classes of mental activity: focused versus relaxed mental activity. The different levels of motor activity achieved with the mouse range from: no motor activity (control condition), semi-automatic motor activity (secondary task with no influence on gameplay), and highly-demanding motor activity (the mouse is used for a primary task, i.e., controlling the position of a virtual character). The performance of the BCI is scrutinized in each condition, as well as subjective preferences of participants.

The remainder of this paper is organized as follows. Section 2 describes related work on the combination of BCI and motor activity. The objectives and experimental protocol of our study are presented in Section 3. The Section 4 details the main results, which are then discussed in Section 5. The paper ends with a general conclusion. 


\section{Related Work}

The joint use of Brain-Computer Interface with other input devices such as a computer mouse has been relatively rarely studied in BCI or VR scientific communities. A recent example is the 2D game "Bacteria Hunt" in which the controllability of a mouse is impaired by considering the level of Alpha band power which is correlated here to relaxed wakefulness [Mühl et al. 2010]. In "AlphaWow", which is based on the famous game World-of-Warcraft, the user's avatar is modified from elf to bear according to the measured level of Alpha activity [Nijholt et al. 2009]. However, the authors of these studies did not specifically compare the use of their setup with and without the manipulation of the mouse.

In the context of virtual environments, two other representative studies report on setups combining joysticks and BCI. First, Kreilinger et al. propose to switch alternatively from joystick to BCI to provide a continuous source of reliable control [Kreilinger et al. 2012]. The two devices were not used simultaneously. The VR entertaining application consists in controlling a car to collect coins and avoid obstacles. A reliability score is computed for both devices, allowing the selection of the most reliable device at each time. The switching between BCI and joystick was found to improve the user experience, allowing the participants to control the car even after loosing control on one of the devices. In another study, Leeb et al. have simultaneously used a joystick and a BCI to control an immersive videogame [Leeb et al. 2013]. The VE, displayed in a CAVE, consisted in a virtual penguin sliding on an icy track. Participants could control the trajectory of the penguin using the joystick. A BCI-based motor imagery paradigm was used to make the penguin occasionally jump to catch virtual fishes. This hybrid interaction scheme was found globally efficient and well appreciated by the participants. But the mutual influence of motor and mental activities was not the main purpose of this paper.

There have been few specific studies on the influence of additional motor activity on the performance of a BCI. First, Lotte et al. reported on a preliminary study evaluating the influence of locomotion and ambulatory motor activities on the performance of a $\mathrm{BCI}$ based on the well-known P300 brain signal [Lotte et al. 2009]. Three different locomotion conditions were tested with different ambulatory motions: sitting, standing and walking. Successful classification of auditory P300 signals could be achieved off-line in every conditions and the best mean classification score was obtained in standing condition. In another study, Gürkök et al. could find that the control of a BCI was not impaired by speaking [Gürkök et al. 2010]. The experiment involved left hand versus right hand motor imagery, with and without speaking. It was found that the (facial) muscular activities involved when speaking had no major impact on the classification results.

\section{Experimental Study}

Our objective is to study if and how a mouse manipulation and its related motor activity could influence the performance of a BCI when interacting with a VE. The experimental apparatus consists of a simple videogame that requires the player to control a mental activity with a BCI and sometimes perform a motor activity by using a mouse. Three complexity levels of motor activity are proposed: $\mathrm{C}_{M O} 1$ (no motor activity), $\mathrm{C}_{M O} 2$ (a semi-automatic motor activity) and $\mathrm{C}_{M O} 3$ (a highly-demanding motor activity). The experiment makes use of a simple two-class BCI measuring the mental activity of the user related to either a relaxed mental activity $\left(\mathrm{C}_{M E}-\mathrm{R}\right)$ or a focused mental activity $\left(\mathrm{C}_{M E}-\mathrm{F}\right)$. Our purpose is to observe the evolution of BCI performance (i.e., the discrimination rate between relaxed and focused mental activities by the BCI system) according to the different levels of motor activity.

Population Eight healthy participants ( 1 female and 7 males, aged from 21 to 28 , mean $=24, \mathrm{sd}=2.1$ ) took part to the experiment. All participants except one were right-handed.

Experimental Apparatus The VE application is a simplified variant of the Pac-Man videogame (Figure 1), designed with the Unity3D game engine (unity3d.com). Videogames are known to be a motivating environment for BCI experiments [Lécuyer et al. 2008]. The user controls a yellow blob in a maze and has to eat pellets to gain points while avoiding contact with two ghosts (two red blobs surrounding the yellow character). The game is controlled both with a mouse and a BCI system (to control the eating of virtual pellets). The experiment was conducted in a room without environmental noise or other source of distraction.

EEG activity was gathered using a g.USBAmp (g.tec company, Austria) amplifier sampled at $512 \mathrm{~Hz}$. Sixteen electrodes were used to gather EEG signals and one additional electrode was used as a reference. A ground electrode was placed on the user's left ear lobe. Electrodes were placed on positions Fp1, Fp2, F7, F8, T7, $\mathrm{T} 8, \mathrm{~F} 3, \mathrm{~F} 4, \mathrm{C} 3, \mathrm{C} 4, \mathrm{P} 3, \mathrm{P} 4, \mathrm{O} 1, \mathrm{O} 2, \mathrm{Pz}$ and $\mathrm{Cz}$ of the international 10-20 system. The reference electrode was placed on position FCz. EEG signals acquisition, processing and classification were done using the OpenViBE software platform [Renard et al. 2010]. The relaxed and focused mental activities of the user were determined using the Alpha activity that is known to increase during relaxation and can be measured on the posterior half of the head [Niedermeyer 2005]. The Alpha activity was quantified using a band power technique [Pfurtscheller and Lopes da Silva 1999]. Band power was computed 16 times per second on a moving window of 1 second (windows could overlap). We have used the same signal-processing pipeline as in [George et al. 2011]. The signal-processing pipeline provides a two-class output that represents the level of relaxation/focus. A negative (respectively positive) value means that the user has a relaxed (respectively focused) mental activity. The absolute value of the classifier output represents the intensity of the mental activity. For example a value of -1.0 would indicate that the user is completely relaxed. A value of 0 would indicate that the user is not especially relaxed or focused.

Experimental Plan Three different levels of motor activity were used with a progressive complexity:

- $\mathrm{C}_{M O}$ 1: no motor activity was executed. The player character was going forward automatically. If the BCI system detected the correct mental activity then the player character could eat pellets and was colored in yellow. Otherwise it was colored in gray.

- $\mathrm{C}_{M 0}$ 2: a semi-automatic motor activity was executed: the participant had to move the mouse continuously in circles. The player character was going forward automatically. If not enough mouse movement was measured then the character appeared blue and was earning fewer points when eating pellets. If the BCI system detected the correct mental activity then the player character could eat pellets and was colored in yellow instead of gray.

- $\mathrm{C}_{M 0}$ 3: a highly-demanding motor activity was executed: the participant had to move the mouse in the same direction as the player character along the square path (Figure 1). The mouse movements actually controlled the motion of the character. The movements had to be at the right speed, to avoid two ghosts following and preceding the character and advancing at a constant speed. If the BCI system detected the correct mental activity and if the player character was well located between the two ghosts, pellets could be eaten and the character was colored in yellow instead of gray. If the player was too slow or too fast and moved behind or in front of the ghosts 
then he could not eat any pellet and the character was colored in red.

Two different mental activities were proposed: $\mathrm{C}_{M E}-\mathrm{R}$ for the Relaxed mental activity and $\mathrm{C}_{M E}-\mathrm{F}$ for the Focused mental activity. In each case the participant was instructed to remain in the given mental state (Relaxed or Focused) during all the trial, in order to collect and eat pellets.

Procedure Each participant was informed about the complete test procedure which comprised a training phase. During the training phase, the user had to watch a static cross on a computer screen and successively relax and concentrate for 30 seconds. The gathered data were used to train the BCI classifier. Then the experiment involved 6 conditions corresponding to the combinations of 3 motor activity conditions $\left(\mathrm{C}_{M O} 1, \mathrm{C}_{M O} 2\right.$, $\left.\mathrm{C}_{M O} 3\right)$ and 2 mental activity conditions $\left(\mathrm{C}_{M E}-\mathrm{R}, \mathrm{C}_{M E}-\mathrm{F}\right)$. The order of presentation of the 6 conditions was randomized. Each trial lasted around 1 minute and 30 seconds. The 6 conditions were passed two times, ending up with $\left(3 \mathrm{C}_{M O}\right) *\left(2 \mathrm{C}_{M E}\right) *$ $(2$ repetitions $)=12$ trials, for a total duration of around 18 minutes.

Collected Data For each trial and each participant, we recorded the BCI classifier output (between -1 and 1) resulting from the signal-processing pipeline. At the end of the experiment, participants had to fill a subjective questionnaire with a 7-point Likertscale. Criteria used were: (1) the ability for the user to generate the appropriate mental activity, (2) the ability of the BCI system to detect the mental activity of the user, (3) the appreciation of game conditions and (4) the fatigue.

\section{Results}

\subsection{Classifier Results}

A mixed between-within subjects ANOVA was conducted to assess the impact of the three motor activities and the two mental activities on the BCI classifier output. We found a significant effect of the mental activity condition on classifier output $(F(1,7)=4.57$, $p=0.005)$. The mean value for the $\mathrm{C}_{M E}-\mathrm{R}$ condition was $-0.17(S D=0.38)$, while the mean value for the $\mathrm{C}_{M E}-\mathrm{F}$ condition was $0.31(S D=0.29)$. A paired-sample t-test shows a significant difference between $\mathrm{C}_{M E}-\mathrm{R}$ and $\mathrm{C}_{M E}-\mathrm{F}$ for all motor activity conditions: $\mathrm{C}_{M 0} 1(t(7)=-3.65, p=0.008), \mathrm{C}_{M 0} 2$ $(t(7)=-4.07, p=0.005)$ and $\mathrm{C}_{M 0} 3(t(7)=-3.02, p=0.019)$. The effect of the motor activity condition was not found significant $(F(2,6)=3.63, p=0.09)$ and no significant interaction between motor activity and mental activity conditions was observed $(F(2,6)=4.57, p=0.06)$. Classifier output for each participant and each motor and mental activity condition are provided in Table 1 . Figure 2 provides the boxplots for all participants and the different combinations of motor and mental activity conditions.

Table 1: Mean classifier output for each participant and for each combination of motor and mental activities. The global mean and standard deviation are also provided.

\begin{tabular}{|c|c|c|c|c|c|c|}
\hline & $\begin{array}{c}\mathrm{C}_{M O} 1 \\
\mathrm{C}_{M E}-\mathrm{R}\end{array}$ & $\begin{array}{c}\mathrm{C}_{M O} 1 \\
\mathrm{C}_{M E}-\mathrm{F}\end{array}$ & $\begin{array}{c}\mathrm{C}_{M O} 2 \\
\mathrm{C}_{M E}-\mathrm{R}\end{array}$ & $\begin{array}{c}\mathrm{C}_{M O} 2 \\
\mathrm{C}_{M E}-\mathrm{F}\end{array}$ & $\begin{array}{c}\mathrm{C}_{M O} 3 \\
\mathrm{C}_{M E}-\mathrm{R}\end{array}$ & $\begin{array}{c}\mathrm{C}_{M O} 3 \\
\mathrm{C}_{M E}-\mathrm{F}\end{array}$ \\
\hline S1 & -0.44 & 0.23 & 0.27 & 0.43 & 0.18 & 0.07 \\
\hline S2 & 0.32 & 0.52 & 0.24 & 0.99 & 0.31 & 0.83 \\
\hline S3 & -0.26 & 0.04 & -0.40 & 0.05 & -0.43 & -0.15 \\
\hline S4 & -0.72 & 0.17 & -1.04 & -0.01 & -0.32 & 0.59 \\
\hline S5 & -0.56 & 0.29 & -0.11 & 0.45 & 0.15 & 0.60 \\
\hline S6 & -0.68 & 0.42 & -0.69 & 0.59 & -0.02 & 0.53 \\
\hline S7 & 0.20 & 0.16 & -0.01 & 0.33 & 0.18 & 0.28 \\
\hline S8 & -0.24 & -0.02 & -0.15 & -0.01 & 0.04 & 0.12 \\
\hline Mean & -0.30 & 0.23 & -0.24 & 0.35 & 0.01 & 0.36 \\
\hline SD & 0.39 & 0.18 & 0.45 & 0.34 & 0.26 & 0.33 \\
\hline
\end{tabular}

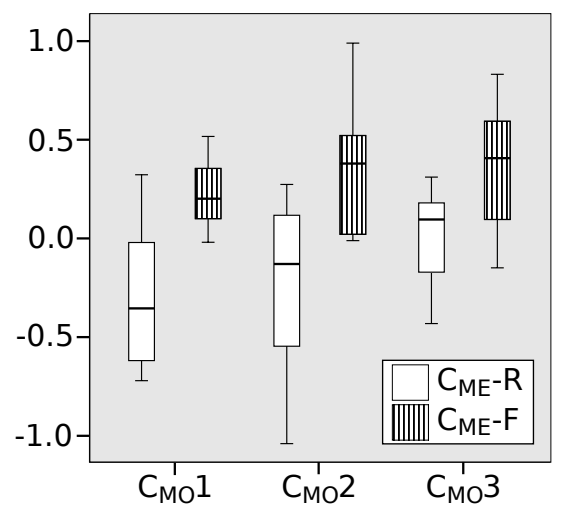

Figure 2: Boxplots of classifier output for each combination of motor and mental activities. They are delimited by the quartile $(25 \%$ / $75 \%)$ of the distribution over all participants. Median is also represented for each boxplot.

\subsection{Questionnaire Results}

Mean values of participants answers to the subjective questionnaire are provided in Table 2. A Friedman test showed no significant difference between the different conditions for all criteria. From the results it seems that whatever the motor activity condition, the participants felt able to generate the appropriate mental activity depending on the instructions. Moreover, the participants globally found that the BCI system correctly detected their mental activity. Results also indicate that participants enjoyed the different conditions of interaction with the game and found it not too tiring.

\section{Discussion}

Considering the analysis of the classifier output, a significant difference was found between the two mental activity conditions $\mathrm{C}_{M E}-\mathrm{R}$ and $\mathrm{C}_{M E}-\mathrm{F}$, whatever the motor activity condition $\left(\mathrm{C}_{M O} 1, \mathrm{C}_{M O} 2\right.$ or $\mathrm{C}_{M O} 3$ ). Thus, the mental activity was correctly detected, even when participants were executing a highly-demanding motor activity with their mouse $\left(\mathrm{C}_{M O} 3\right)$. Participants were able to adapt their mental activity independently of any motor activity, showing the effectiveness of the BCI-based interaction proposed in this study.

Looking at Table 1, we can observe that the average values between $\mathrm{C}_{M E}-\mathrm{R}$ and $\mathrm{C}_{M E}-\mathrm{F}$ are closer when the motor activity is highly demanding $\left(\mathrm{C}_{M O} 3\right)$. It suggests that the $\mathrm{BCI}$ is less effective in $\mathrm{C}_{M O} 3$ than in the other motor activity conditions. The mean classification value for the combination of the conditions $\mathrm{C}_{M O} 3$ and $\mathrm{C}_{M E}-\mathrm{R}$ suggests that participants had some difficulties executing a highly-demanding motor activity while trying to achieve a relaxed mental activity. However, no significant difference was found in the subjective questionnaire for the subjective ability to generate the appropriate activity. This suggests that the user subjective perception of the BCI-based interaction was not considerably affected by the motor activity condition.

Further investigation on the combination of BCI and input devices could confirm our results for introducing motor activity while using a BCI within a VE. Future work could include more applications of simple BCI as additional interfaces for controlling virtual environments. Other mental activities, or BCI paradigms, could also be studied as well as other VR interfaces. Additional experiments could also be performed within a 3D virtual environment with a higher immersion or when allowing the users to stand or walk during the experiment. 
Table 2: Mean values (and standard deviations in brackets) of answers to the subjective questionnaire, with respect to the conditions. The criteria are: $(C 1)$ the ability for the user to generate the appropriate mental activity, $(C 2)$ the ability of the BCI system to detect the mental activity, (C3) the appreciation of game, and (C4) the fatigue. Mental activities are differentiated for the two first criteria (relaxed and focused mental activity in this order).

\begin{tabular}{|c|c|c|c|}
\hline & $\mathrm{C}_{M O} 1$ & $\mathrm{C}_{M O}$ & $\mathrm{C}_{M O}$ \\
\hline C1: Relaxed/Focused & $5.62(0.92) / 4.62(0.92)$ & $4.87(1.36) / 4.87(1.13)$ & $4.37(1.69) / 5.62(0.74)$ \\
\hline C2: Relaxed/Focused & $4.87(1.89) / 4.87(1.64)$ & $4.5(1.77) / 4.75(1.83)$ & $4.62(1.69) / 4.87(1.46)$ \\
\hline C3 & $5.25(1.39)$ & $4.25(1.58)$ & $5.62(1.77)$ \\
\hline $\mathrm{C} 4$ & $4.87(1.13)$ & $5(1.41)$ & $4.87(1.89)$ \\
\hline
\end{tabular}

\section{Conclusion}

In this paper, we have presented a study on the influence of motor activity and mouse manipulation on the performance of a simple BCI for controlling a virtual environment. Different levels of motor activity were tested: no motor activity, a semi-automatic motor activity and a highly-demanding motor activity. Our results show that our simple 2-class BCI could be successfully used in each case, and thus even in presence of a highly demanding motor activity (when the users were carefully controlling the 2D position of a virtual character with the mouse). Moreover, the participants subjectively felt that they were able to control their mental activity in all cases, and enjoyed the entertaining and hybrid mouse/BCI interaction with the virtual environment. These results pave the way to future experimental studies with more complex mental and motor activities, but also to novel 3D interaction paradigms that could mix $\mathrm{BCI}$ and other input devices for VR and videogame applications.

\section{Acknowledgements}

This work was supported by the administrative region of Brittany and the French National Research Agency within Homo Textilus project (grant ANR-11-SOIN-007).

\section{References}

Allison, B., Graimann, B., And GrÄSER, A. 2007. Why use a BCI if you are healthy? In ACE Workshop-BCI and Games, $7-11$.

George, L., Lotte, F., Abad, R. V., And Lécuyer, A. 2011. Using scalp electrical biosignals to control an object by concentration and relaxation tasks: design and evaluation. In the IEEE Int. Conf. EMBS, 6299-6302.

GÜrkÖK, H., Poel, M., AND ZwIERs, J. 2010. Classifying motor imagery in presence of speech. In the Int. Joint Conf. on Neural Networks, 1235-1242.

Kreilinger, A., Kaiser, V., Breitwieser, C., Williamson, J., Neuper, C., AND Müller-Putz, G. 2012. Switching between manual control and brain-computer interface using long term and short term quality measures. Frontiers in Neuroscience 5, 147.

Lécuyer, A., Lotte, F., Reilly, R., Leeb, R., Hirose, M., AND SLATER, M. 2008. Brain-computer interfaces, virtual reality, and videogames. Computer 41, 10, 66-72.

LEE, H., AND CHOI, S. 2003. PCA+HMM+SVM for EEG pattern classification. In Seventh International Symposium on Signal Processing and Its Applications, vol. 1, 541-544.

Leeb, R., Lancelle, M., Kaiser, V., Fellner, D. W., And Pfurtscheller, G. 2013. Thinking penguin: Multi-modal brain-computer interface control of a VR game. IEEE Trans. on Comp. Intelligence and AI in Games.

Lotte, F., Fujisawa, J., Touyama, H., Ito, R., Hirose, M., AND LÉCUYER, A. 2009. Towards ambulatory brain-computer interfaces: A pilot study with $\mathrm{P} 300$ signals. In Int. Conf. on ACE Technology, 336-339.

Lotte, F., Faller, J., Guger, C., Renard, Y., PfuRTSCHEller, G., LÉCUYER, A., AND LEEB, R. 2013. Combining BCI with virtual reality: Towards new applications and improved BCI. In Chapter in Towards Practical Brain-Computer Interfaces, B. Allison and et al., Eds., 197-220.

McFarland, D., AND WolpaW, J. 2011. Brain-computer interfaces for communication and control. Communications of the ACM 54, 5, 60-66.

MÜHL, C., GÜRKÖK, H., Bos, D. P.-O., Thurlings, M., Scherffig, L., Duvinage, M., Elbakyan, A., Kang, S., Poel, M., AND HeYlen, D. 2010. Bacteria hunt: Evaluating multi-paradigm BCI interaction. Journal Multimodal User Interfaces 4, 11-25.

NIEDERMEYER, E. 2005. The normal EEG of the waking adult. Electroencephalography: Basic principles, clinical applications, and related fields, 167.

Nijholt, A., Reuderink, B., And Bos, D. O. 2009. Turning shortcomings into challenges: Brain-computer interfaces for games. In Intelligent Technologies for Interactive Entertainment, 153-168.

Pfurtscheller, G., ANd Lopes da Silva, F. 1999. Eventrelated EEG/MEG synchronization and desynchronization: basic principles. Clinical Neurophysiology 110, 11, 1842-1857.

Renard, Y., Lotte, F., Gibert, G., Congedo, M., Maby, E., Delannoy, V., Bertrand, O., ANd LÉCUYeR, A. 2010. OpenViBE: An open-source software platform to design, test, and use brain-computer interfaces in real and virtual environments. Presence: Teleoperators and Virtual Environments 19, 3-53. 